\title{
Nuevo método de evaluación del riesgo de erosión hídrica en operaciones forestales basado en la variabilidad de las precipitaciones
}

\author{
A new method of assessing water erosion risk in forest operations based on rainfall variability
}

\author{
Juan Contreras ${ }^{a}$, Carlos A Bonilla ${ }^{b *}$, Juan Troncoso ${ }^{a}$ \\ a Pontificia Universidad Católica de Chile, Departamento de Economía Agraria, Vicuña Mackenna 4860, Macul, Santiago, Chile, \\ tel.: 56-2-3544180, fax: 56-2-6865727. \\ *Autor de correspondencia: ${ }^{\text {b }}$ Pontificia Universidad Católica de Chile, Departamento de Ingeniería Hidráulica y Ambiental, \\ Vicuña Mackenna 4860, Macul, Santiago, Chile, tel.: 56-2-3544227, fax: 56-2-3545868, cbonilla@ing.puc.cl
}

\begin{abstract}
SUMMARY
Water erosion has been a major environmental problem associated to forest harvesting operations in humid and temperate climates. The incorporation of this factor in the decision making process has been complex due to the high variability observed in rainfall intensity and magnitude. This paper presents a methodology for incorporating the temporal variability of rainfall in the planning of forest operations. We used a monthly index based on the R-RUSLE erosivity factor. This index allows knowing the most likely value of soil loss and associating a confidence level. Thus, the forest planner has an estimate of erosion rates related to the operations, and an index that reflects the rainfall variability. With this methodology it is possible to identify, for a given level of soil loss, those months in which there is a higher uncertainty. This methodology was applied to a pine plantation in Santa Barbara, Biobío Region, Chile, where the rainfall erosivity ranges from $16 \mathrm{MJ} \mathrm{mm} \mathrm{ha}^{-1} \mathrm{~h}^{-1}$ to $271 \mathrm{MJ} \mathrm{mm} \mathrm{ha}^{-1} \mathrm{~h}^{-1}$ depending on the month of the year.
\end{abstract}

Key words: forest operation planning, Pinus radiata, R-RUSLE, rainfall erosivity, water erosion.

\section{RESUMEN}

La erosión hídrica es uno de los principales problemas ambientales asociados a las faenas forestales en climas húmedos y templados. La incorporación de este factor en el proceso de toma de decisiones ha sido compleja dada la alta variabilidad observada en las precipitaciones, su intensidad y magnitud. Este estudio presenta una metodología para incorporar la variabilidad temporal de las precipitaciones en la planificación de operaciones forestales. Para ello se elaboró un nuevo índice mensual basado en el factor de erosividad $R$-RUSLE. Este índice permite conocer el valor más probable de pérdida de suelo y asociarle un nivel de confianza. De esta forma, el planificador forestal dispone de una estimación de las tasas de erosión asociadas a las faenas, y de un índice que refleja la variabilidad climática de las lluvias. Con esta metodología es posible identificar, para un nivel dado de pérdida de suelo, aquellos meses en los cuales existe un mayor grado de incertidumbre asociada. Esta metodología fue aplicada en una plantación de Pinus radiata en la localidad de Santa Bárbara, región del Biobío, Chile, donde se observaron variaciones en la erosividad de las precipitaciones desde $16 \mathrm{MJ} \mathrm{mm} \mathrm{ha}{ }^{-1} \mathrm{~h}^{-1}$ hasta $271 \mathrm{MJ} \mathrm{mm} \mathrm{ha}^{-1} \mathrm{~h}^{-1}$ dependiendo del mes del año.

Palabras clave: erosión hídrica, erosividad, Pinus radiata, planificación forestal, R-RUSLE.

\section{INTRODUCCIÓN}

Uno de los principales impactos ambientales asociados a las faenas forestales es la pérdida de suelo (Gayoso y Alarcón 1999). Producto de ello, se han desarrollado diversos estudios para medir o estimar los efectos de la erosión hídrica en terrenos forestales (Oyarzún 1993, Peña et al. 1993, Hartanto et al. 2003, Fernández et al. 2004, Croke y Nethery 2006). También se han elaborado modelos de predicción, destacando entre ellos por su amplia utilización la ecuación universal de pérdida de suelo USLE (Wischmeier y Smith 1978) y su versión revisada RUSLE (Renard et al.
1997). En RUSLE, los efectos de los principales factores involucrados en la pérdida de suelo se evalúan mediante la siguiente ecuación:

$$
A=R \cdot K \cdot L \cdot S \cdot C \cdot P
$$

donde $A$ es la pérdida de suelo $\left(\mathrm{Mg} \mathrm{ha}^{-1} \mathrm{año}^{-1}\right), R$ es el factor de erosividad de las precipitaciones (MJ $\mathrm{mm} \mathrm{ha}^{-1} \mathrm{~h}^{-1}$ año-1), $K$ es el factor de erodabilidad del suelo ( $\mathrm{Mg}$ ha h ha ${ }^{-1}$ $\mathrm{MJ}^{-1} \mathrm{~mm}^{-1}$ ), $L$ y $S$ son los factores de longitud y pendiente de la ladera respectivamente, $C$ es el factor de cobertura o manejo y $P$ es el factor de prácticas de conservación. Los factores $L, S, C$ y $P$ son adimensionales. 
Con el fin de facilitar la aplicación de USLE y RUSLE en el ámbito forestal, se han modificado algunos de sus parámetros originales. Así por ejemplo Dissmeyer y Foster (1983) subdividen el factor de cobertura o manejo en nueve sub-factores que se asocian entre otros, a los efectos de las faenas forestales e incorporan en ellos el factor de prácticas de conservación. En otro estudio, Özhan et al. (2005) proponen valores para el producto de la combinación de los factores de cobertura y manejo y de prácticas de conservación en ecosistemas forestales. En las investigaciones antes descritas, los factores que determinan el proceso de erosión son parametrizados a base de datos históricos. En esta parametrización, la variabilidad espacial y temporal de los factores de RUSLE se elimina y, por tanto, no se incorpora en la cuantificación de las pérdidas de suelo. El no considerar esta variación, y trabajar sólo con los valores medios, puede hacer que la pérdida de suelo observada en terreno sea muy distinta de la estimada con el modelo (Wang et al. 2002). En un intento por incorporar dicha variabilidad, Dissmeyer y Foster (1983) descomponen el factor de erosividad de la lluvia R-RUSLE en promedios mensuales, y proponen evaluar el riesgo de erosión mediante dicha desagregación. Si bien esto permite una estimación más realista al calcular una tasa de erosión mensual, no se informa sobre la variabilidad climática asociada a las precipitaciones en cada uno.

En consecuencia, el objetivo de este estudio es presentar una metodología que permite incorporar, en la planificación de cosecha, el efecto de la variabilidad climática de las precipitaciones mensuales y su relación con el proceso de erosión. De esta forma, el planificador forestal cuenta con una herramienta adicional para el diseño de un plan de cosecha ambientalmente adecuado.

\section{MÉTODOS}

La metodología propuesta consistió en un proceso de dos etapas. En primer lugar, se calculó el promedio y la desviación estándar de las erosividades mensuales de acuerdo a la metodología descrita para RUSLE (Dissmeyer y Foster 1983). El factor de erosividad de las precipitaciones $(R)$ se calculó a escala mensual para considerar la variación estacional de las precipitaciones (Lu et al. 2003). Luego, se estableció un ponderador de variabilidad para sumar el promedio y la desviación estándar de la erosividad mensual y así construir el índice de erosión. En el índice de erosión, se emplearon los factores de RUSLE adaptados a terrenos forestales descritos por Dissmeyer y Foster (1983).

De esta forma, el índice de erosión se calculó como la suma ponderada del promedio y la desviación estándar de la erosividad mensual de la siguiente manera:

$$
\mathrm{I}_{\mathrm{i}}=\left[\mathrm{R}_{\mathrm{i}} \cdot \alpha+\mathrm{S}_{\mathrm{i}} \cdot(1-\alpha)\right] \cdot \mathrm{K} \cdot \mathrm{L} \cdot \mathrm{S} \cdot \mathrm{C}
$$

donde $I_{i}$ es el índice de erosión mensual del mes $i, R_{i}$ es el factor de erosividad promedio del mes $i, S_{i}$ es la desviación estándar del factor de erosividad del mes $i$ y $\alpha$ es el ponderador de variabilidad del factor de erosividad que va de 0 a 1 . Los parámetros $K, L, S$ y $C$ corresponden a los factores de erodabilidad, longitud de la ladera, pendiente de la ladera y cobertura o manejo, respectivamente. El factor $C$ se calculó con los nueve sub-factores descritos por Dissmeyer y Foster (1983), incluyendo en este el efecto del factor $P$.

El ponderador $\alpha$ se define de acuerdo al nivel de aversión al riesgo del planificador forestal y es constante para todos los meses del año. Un valor $\alpha=1$ cancela el segundo término del paréntesis de la ecuación [2] y hace que el índice de erosión mensual $I_{i}$ sea igual al valor de erosión calculado directamente con RUSLE. De esta forma, el planificador pone todo el peso de la estimación en el valor promedio de las erosividades. Por el contrario, $\alpha=0$ anula el término $R_{i}$ de la ecuación [2] y hace que el índice de erosión mensual dependa sólo de la variabilidad (desviación estándar) sin importar el valor de la tendencia central (promedio). Cualquier otro valor de $\alpha$ entre 0 y 1 hace que el índice de erosión considere tanto la tendencia central como la variabilidad de las erosividades.

La metodología se aplicó a una plantación de Pinus radiata D. Don en la localidad de Santa Bárbara, región del Biobío, Chile. El registro de precipitaciones se obtuvo de la estación meteorológica Quilaco, dependiente de la Dirección General de Aguas, y ubicada a $6 \mathrm{~km}$ de la plantación ( $37^{\circ} 41^{\prime} \mathrm{S}, 72^{\circ} 00^{\prime}$ O, $225 \mathrm{~m}$ s.n.m.). Se emplearon 17 años de registro (de 1976 a 1992), siendo 15 años un valor mínimo recomendado para el cálculo del factor de erosividad (Foster et al. 2003). El factor de erosividad se calculó de acuerdo a la metodología descrita por Renard et al. (1997). Para cada tormenta se determinó el valor de $E I_{30}$, el cual corresponde al producto de la energía aportada por las gotas de lluvia en la tormenta, $E$, y la intensidad máxima registrada en 30 min durante la tormenta, $I_{30}$. De acuerdo a la metodología original, no se incluyeron en el cálculo tormentas de menos de $12,7 \mathrm{~mm}$, a menos que se hubiesen registrado intensidades iguales o superiores a $6,4 \mathrm{~mm}$ en $15 \mathrm{~min}$ durante la misma.

Para el factor de erodabilidad $K$ se empleó un valor de $0,09 \mathrm{Mg}$ ha h ha- $\mathrm{MJ}^{-1} \mathrm{~mm}^{-1}$, y para el caso del producto de los factores de longitud y pendiente de la ladera se asignó un valor de 3,59 (120 m de longitud y $12 \%$ inclinación). Ambos valores se obtuvieron de Peña et al. (1993). Los valores del factor cobertura $C$ se asignaron de acuerdo a Dissmeyer y Foster (1983), 1 para el caso de suelo descubierto y 0,3 para la condición con desechos de la cosecha.

\section{RESULTADOS}

En la figura 1 se observa una alta variabilidad en la erosividad a lo largo de los meses del año en respuesta a las características de las lluvias en Quilaco. Las erosividades más altas se registran desde mayo hasta agosto, 

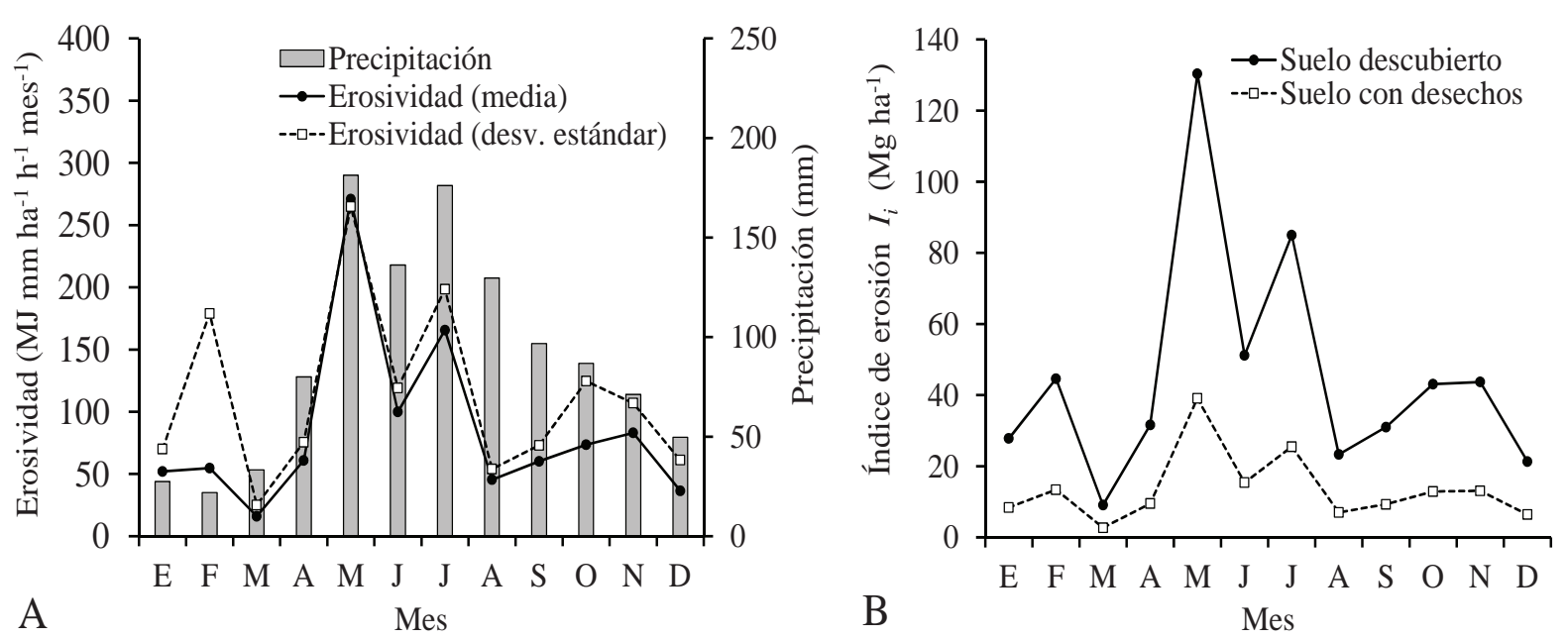

Figura 1. Relación entre la precipitación y el índice de erosión. A) Precipitación promedio y la media y desviación estándar de la erosividad mensual en estación Quilaco. B) Factor de erosividad mensual ( $\left.\mathrm{MJ} \mathrm{mm} \mathrm{ha}^{-1} \mathrm{~h}^{-1}\right)$ y el índice de erosión $I_{i}\left(\mathrm{Mg} \mathrm{ha}^{-1}\right)$ calculado para un sistema de cosecha tradicional en Quilaco $(\alpha=0,7, C=1$ para suelo descubierto, y $C=0,3$ para suelo con desechos).

Relationship between precipitation and the erosion index. A) shows the average rainfall and the mean and standard deviation of monthly erosivity at Quilaco station. B) shows the monthly erosivity factor (MJ mm ha $\left.\mathrm{h}^{-1}\right)$ and the erosion index Ii $\left(\mathrm{Mg} \mathrm{ha}^{-1}\right)$ calculated for a traditional harvesting system at Quilaco ( $\alpha=0.7, \mathrm{C}=1$ for bare soil, and $\mathrm{C}=0.3$ for soil with residues).

coincidiendo con los meses más lluviosos. Un caso particular, y relevante para el cálculo del índice de erosión es lo observado en los meses de enero y febrero. En ambos meses la erosividad promedio es similar, pero la desviación estándar es considerablemente mayor en febrero (179 MJ mm ha- $\mathrm{h}^{-1}$ versus $70 \mathrm{MJ} \mathrm{mm} \mathrm{ha}^{-1} \mathrm{~h}^{-1}$ ). Esto hace que el índice de erosión sea mucho más alto en febrero aun cuando registra casi la misma erosividad promedio que el mes de enero.

En el cálculo del índice de erosión $I_{i}$ se empleó un $\alpha=0,7 ; \mathrm{y}$, salvo en el caso del factor de cultivo $C$, el resto de los factores de RUSLE se asumieron constantes para la condición de suelo descubierto y con desechos de cosecha. En las dos condiciones de suelo evaluadas, se observa que los índices de erosión más altos se asocian a mayor variabilidad en la erosividad $\left(S_{i}\right)$ y, por ende, tienen un grado de incertidumbre mayor. Por el contrario, índices de erosión pequeños se asocian a desviaciones estándar pequeñas. De acuerdo a estos resultados, y para esta localidad, las faenas forestales que más afectan el suelo se deberían concentrar en el mes de marzo. En este mes es posible reducir la protección del suelo con menor riesgo, ya que presenta el menor índice de erosión a consecuencia de una erosividad menor, en magnitud y variabilidad. Por el contrario, las labores de cosecha deben ser las mínimas en el mes de mayo, si lo que se quiere es tener el menor impacto negativo en la conservación del suelo.

\section{DISCUSIÓN}

En el ámbito de la planificación, el índice de erosión permite identificar de mejor forma el momento más ade- cuado para la implementación de las distintas faenas forestales y la necesidad de implementar o no técnicas de protección de suelo. De este modo, la decisión de minimizar las pérdidas de suelo se hace más integral al considerar los promedios mensuales de erosividad y sus desviaciones. En consecuencia, si para implementar una labor se debe elegir entre dos meses con promedios de erosividad similares pero desviaciones estándar distintas, se debería elegir el mes con el índice de erosión más pequeño. Sin embargo, tanto los promedios como las desviaciones suelen ser distintas. En ese caso, la metodología descrita incorpora el valor que asigna el planificador forestal a la magnitud e incertidumbre de las erosividades mediante el ponderador de variabilidad $(\alpha)$. El ponderador $\alpha$ opera como un indicador de aversión al riesgo; y el cálculo del índice de erosión está determinado por la mayor importancia otorgada al promedio o la desviación de las erosividades.

Además de la metodología propuesta en este estudio, la variabilidad en las pérdidas de suelo también puede ser incorporada en otros factores de RUSLE. Esto depende de la disponibilidad de información y del criterio del planificador forestal. A partir de su experiencia sobre el sitio, el planificador puede ponderar de forma distinta cada factor de RUSLE. Por ejemplo, dado que existen pocos estudios relacionados con el comportamiento factor $K$ en terrenos forestales, en esta evaluación se siguió el procedimiento de Wischmeier y Smith (1978). Sin embargo, en el caso de disponer de mediciones y distribución espacial de este factor en el sitio, sería posible incorporar su variabilidad en la ecuación [2]. Caso distinto son los factores de longitud y pendiente de la ladera $(L$ y $S)$, sobre los cuales hay más aplicaciones gracias al empleo de los sistemas 
de información geográfica (SIG) y otras herramientas de representación espacial (Pandey et al. 2007, García y Giménez 2010).

\section{CONCLUSIONES}

La incorporación de la variabilidad climática a la estimación de las tasas de erosión a través de la erosividad de las precipitaciones permite al planificador de operaciones forestales realizar una estimación más confiable del impacto de las faenas sobre la pérdida de suelo. Esta variabilidad se puede incorporar mediante el cálculo de un índice de erosión, el cual considera no sólo el valor medio de las erosividades, sino también su variación. Esto permite programar de forma más realista las actividades de cosecha bajo un criterio de conservación de suelo. La metodología aquí descrita constituye un procedimiento simple y efectivo para evaluar ambientalmente diferentes alternativas de planificación tales como la reasignación de sistemas de cosecha y el manejo de desechos.

\section{REFERENCIAS}

Croke J, M Nethery. 2006. Modeling runoff and soil erosion in logged forests: Scope and Application of some existing models. Catena 67: 35-49.

Dissmeyer G, G Foster. 1983. A guide for predicting sheet and rill erosion on forest land. USDA Forest Service. p. 7-19.

Fernández C, J Vega, J Gras, T Fonturbel, P Cuiñas, E Dambrine, M Alonso. 2004. Soil erosion alters Eucalyptus globulus clearcutting: differences between logging flash disposal treatments. Forest Ecology and Management 195: 85-95.

Foster G, D Yoder, G Weesies, D McCool, K McGregor, R Bingner. 2003. Draft user's guide, Revised Universal Soil Loss Equation Version 2 (RUSLE2). Washington, DC. USDA-Agricultural Research Service. 77 p.

García J, M Giménez. 2010. Estimation of slope length value of RUSLE factor L using GIS. Journal of Hydrologic Engineering 15(9): 714-717.

Gayoso J, D Alarcón. 1999. Guía de conservación de suelos forestales. Proyecto de certificación del manejo forestal en las Regiones Octava, Décima y Duodécima. Valdivia,
Chile. INFOR. 96 p.

Hartanto H, R Prabhu,A Widayat, CAsdak. 2003. Factors affecting runoff and soil erosion: plot-level soil loss monitoring for assessing sustainability of forest management. Forest Ecology and Management 180: 361-374.

Lu H, I Prosser, C Moran, J Gallant, G Priestley, J Stevenson. 2003. Predicting sheetwash and rill erosion over the Australian continent. Australian Journal of Soil Research 41: 1037-1062.

Oyarzún, C. 1993. Evaluación del modelo USLE para predecir pérdidas de suelo en áreas forestales de la Cuenca del Río Bío-Bío. Bosque 14(1): 45-54.

Özhan S, A Balci, N Özyuvaci, A Hizal, F Gökbulak, Y Serengil. 2005. Cover and management factors for the Universal Soil-Loss Equation for forest ecosystems in the Marmara region, Turkey. Forest Ecology and Management 214: 118123.

Pandey A, V Chowdary, B Mal. 2007. Identification of critical erosion prone areas in the small agricultural watershed using USLE, GIS and remote sensing. Water Resources Management 21: 729-746.

Peña L, P Carrasco, M Figueroa, C Oyarzún, B Lo Cascio. 1993. Pérdidas por erosión hídrica en suelos agrícolas y forestales de la cuenca del río Bío-Bío. In Serie Propuestas de Ordenamiento Vol. 4. Seminario Internacional Elementos Cognoscitivos Sobre el Recurso Suelo y Consideraciones Generales Sobre el Ordenamiento Agroforestal. EULA. Ministerio Degli Affari Esteri Della República Italiana/ Ministerio de Planificación y Cooperación de la República de Chile. Concepción: p. 47-60.

Renard, K, G Foster, G Weesies, D McCool, D Yoder. 1997. Predicting soil erosion by water: A guide to conservation planning with the revised universal soil loss equation (RUSLE). U.S. Department of Agriculture, Agriculture Handbook No.703, Washington DC. 407 p.

Wang G, G Gertner, V Singh, S Shinkareva, P Parysow, A Anderson. 2002. Spatial and temporal prediction and uncertainty of soil loss using the revised universal soil loss equation: a case study of the rainfall-runoff erosivity $\mathrm{R}$ factor. Ecological Modelling 153: 143-155.

Wischmeier W, D Smith. 1978. Predicting rainfall erosion losses - a guide to conservation planning. Washington DC., USA. U.S. Department of Agriculture. 58 p. (Agriculture Handbook No. 537). 